\title{
Avaliação das precipitações geradas pelo modelo climático regional ETA-HadGEM2-ES para o Estado de Sergipe
}

\begin{abstract}
Evaluation of the precipitation generated by the regional climate model ETA-HadGEM2-ES for
\end{abstract} the State of Sergipe

\author{
M. A. S. Cruz ${ }^{*} ;$ P. V. M. Mota ${ }^{1} ;$ R. Aragão ${ }^{2} ;$ R. O. F. Rocha ${ }^{1}$ \\ ${ }^{1}$ Embrapa Tabuleiros Costeiros, CEP:49032-280, Aracaju-SE, Brasil \\ ${ }^{2}$ Centro de Tecnologias e Recursos Naturais, Universidade Federal de Campina Grande, CEP: 58429-900, Campina \\ Grande-PB, Brasil
}

*marcusascruz@gmail.com

(Recebido em 19 de junho de 2017; aceito em 30 de setembro de 2017)

\begin{abstract}
O modelo climático regional ETA-HadGEM2-ES tem sido utilizado como base para diversos estudos hidrológicos e climatológicos em território nacional, no entanto ainda há pouca avaliação de sua condição de representatividade das chuvas na região Nordeste, principalmente em sua faixa litorânea, que apresenta grande complexidade nos fenômenos atuantes. Este estudo utilizou dados mensais de chuva medidos em campo, complementados por bases de dados interpolados visando avaliar a condição de representatividade da sazonalidade das precipitações geradas pelo RCM sobre as três regiões climáticas do estado de Sergipe: Agreste, Semiárido e Litoral Úmido. A performance do RCM foi avaliada partir do coeficiente de correlação de Pearson e da raiz do erro médio quadrático (RMSE). Os resultados apontaram para uma baixa capacidade de representação das chuvas médias mensais pelo modelo regional, com subestimação dos volumes totais e baixos coeficientes de correlação, variando de 0,019 a 0,143 para as regiões avaliadas, além de altos valores de RMSE: 58,7 a 143,8 mm, com pequena melhora na região Semiárida. Tais resultados indicam uma possível propagação do erro proveniente do modelo global HadGEM2-ES para o ETA não apresentando nesta faixa do litoral brasileiro uma melhora significativa na representação dos sistemas climáticos atuantes. Palavras-chave: mudanças climáticas, Nordeste, sazonalidade
\end{abstract}

The ETA-HadGEM2-ES regional climate model has been used as the basis for several hydrological and climatological studies in the national territory, however, there is still little evaluation of its rainfall representativeness in the Northeastern region, mainly in its coastal range, which presents large complexity in the climatic systems. This study used monthly rainfall data measured in the field, complemented by interpolated databases to evaluate the seasonality of rainfall generated by the RCM in the three climatic regions of the state of Sergipe: Agreste, Semi-Arid and Wet Litoral. The RCM performance was evaluated from the Pearson correlation coefficient and the root mean square error (RMSE). The results pointed to a low capacity of representation of the average monthly rainfall by the regional model, with underestimation of the total volumes and low correlation coefficients, ranging from 0.019 to 0.143 for the regions evaluated, in addition to high RMSE values: 58.7 to $143.8 \mathrm{~mm}$, with little improvement in the semi-arid region. These results indicate a possible propagation of the error from the global model HadGEM2-ES for the ETA, not showing in this range of the Brazilian coast a significant improvement in the representation of the active climatic systems.

Keywords: climate change, Northeastern, seasonality

\section{INTRODUÇÃO}

A avaliação dos efeitos das alterações no clima sobre uma determinada região tem sido recentemente objeto de muitos estudos, principalmente em decorrência dos impactos que as mudanças nos regimes e quantidades de chuva e temperaturas começam a gerar sobre os sistemas econômicos, sociedade e meio ambiente [1].

Em escala mundial, os modelos globais de circulação climática (GCM) representam de forma satisfatória o comportamento médio de variáveis climáticas, no entanto, estudos têm demonstrado deficiências na representação dos processos ao considerar regiões menores, uma vez que a resolução espacial de avaliação é da ordem de $200 \mathrm{~km}$ [2]. Assim, institutos de estudos de clima de 
diversos países têm adotado procedimentos de redução de escala por meio da aplicação de modelos regionais de clima (RCM), denominados downscaling dinâmico, que utilizam as condições de contorno geradas pelos GCMs, permitindo a redução das células de estudo para valores médios da ordem de $20 \mathrm{~km}$ [3]. Desta forma, é esperado que os RCM possam melhorar os resultados das simulações dos GCM, capturando a amplitude de um fenômeno com maior precisão [4].

Diversos autores apresentaram tentativas de aplicação de modelos regionais de previsão climática sobre a América do Sul [5, 6, 7, 8], com resultados que apontaram para a importância da aplicação do downscaling dinâmico visando melhorar a qualidade da previsão de precipitação de modelos globais utilizados como forçante lateral. Segundo Sun et al. (2005) [15] o modelo Regional Spectral Model (RSM) aninhado ao European-Hamburg Model (MCGA ECHAM4.5) mostrou uma melhor representação da variabilidade das chuvas na região Nordeste do Brasil. Em estudo [16] desenvolvido em três regiões brasileiras, Nordeste, Sudeste e Sul, foi verificado que o modelo climático regional RegCM3, aninhado ao modelo global CPTEC/COLA mostrou desempenho superior na previsão da chuva sazonal, sendo mais próxima da observada se comparada a prevista pelo CPTEC/COLA.

O RCM ETA-HadGEM2 ES, tem sido utilizado como base para diversos estudos hidrológicos e climatológicos em território nacional [9], no entanto ainda há pouca avaliação de sua condição de representatividade das chuvas na região Nordeste, principalmente em sua faixa litorânea, que apresenta grande complexidade nos fenômenos atuantes. Em geral, os principais sistemas meteorológicos que governam o regime de chuvas na região são: Zona de Convergência Intertropical (ZCIT) sobre o oceano Atlântico, Vórtices Ciclônicos de Altos Níveis (VCAN); Frentes Frias e Ondas de Leste. Além destes mecanismos, há também a atuação das linhas de Instabilidade (LI), dos Complexos Convectivos de Mesoescala (CCM), e do efeito das brisas marítima e terrestre na precipitação [10]. Além disso, a forma de atuação destes sistemas depende de uma combinação de forças de eventos El Niño-Oscilação Sul (ENOS); Temperatura da superfície do mar (TSM) na bacia do oceano Atlântico, Ventos Alísios, Pressão ao Nível do Mar (PNM).

Considerando-se uma abordagem por bioma na região Nordeste, o Primeiro Relatório de Avaliação Nacional do Painel Brasileiro de Mudanças Climáticas [1] forneceu os resultados da compilação das projeções de modelos de mudança climática para cenários futuros de temperatura e precipitação, indicando, por exemplo, projeções de redução nas chuvas da ordem de 35\%, na Mata Atlântica da região Nordeste, e de até 50\% na Caatinga até o ano de 2100.

Este estudo busca contribuir para a avaliação da condição de representatividade da sazonalidade das chuvas pelo modelo regional climático ETA-HadGEM2 ES no estado de Sergipe visando contribuir para a previsão de cenários futuros de precipitação de médio e longo prazos.

\section{MATERIAL E MÉTODOS}

A região de estudo (Figura 1) foi o estado de Sergipe, situado na região Nordeste do Brasil, entre as coordenadas geográficas $9^{\circ} 31^{\prime} \mathrm{S}, 38^{\circ} 14^{\prime} \mathrm{W}$ e $11^{\circ} 33^{\prime} \mathrm{S}, 36^{\circ} 25^{\prime} \mathrm{W}$ Datum SIRGAS2000, composto por 75 municípios, que abrangem uma área de aproximadamente $22 \mathrm{mil} \mathrm{km}^{2}$ [11]. Abriga uma população total de 2,3 milhões de habitantes, com grande concentração na região metropolitana de Aracaju (cerca de 1,0 milhão de hab.). As principais atividades econômicas desenvolvidas são agricultura, com destaque para cana de açúcar, milho, mandioca, frutíferas (coco e laranja), criação de gado e exploração mineral, principalmente petróleo, gás natural e potássio.

O menor Estado da Federação caracteriza-se pela presença bem definida de três regiões climáticas: Semiárido - em sua porção extremo oeste, com precipitações anuais médias entre 500 e $800 \mathrm{~mm}$; Agreste - região intermediária do Estado, com precipitações médias de 900 a $1200 \mathrm{~mm}$ ao ano e Litoral Úmido, junto ao oceano Attântico, com precipitações médias anuais superiores a $1600 \mathrm{~mm}$. O período chuvoso ocorre de abril a agosto com concentração nos meses de maio, junho e julho [11]. 


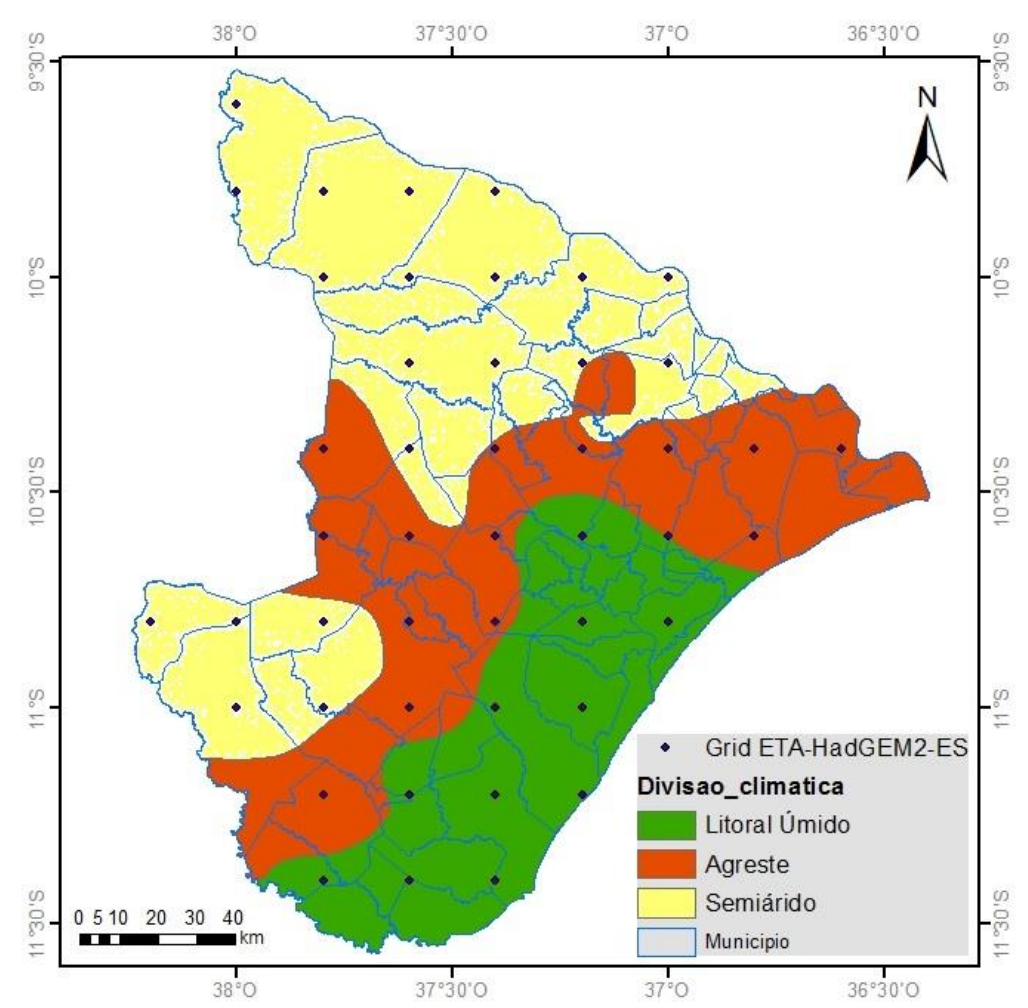

Figura 1: Localização do estado de Sergipe com indicação das três regiões climáticas e os pontos da grade do modelo climático regional ETA-HadGEM2-ES.

O modelo climático regional ETA vem sendo utilizado pelo Instituto Nacional de Pesquisas Espaciais (INPE) desde 1997 para previsão climática. Neste estudo foi utilizada a versão ETA HadGEM2-ES, que utiliza os resultados do GCM HadGEM2-ES desenvolvido pelo Hadley Centre for Climate Change da Inglaterra como condições de contorno. O RCM ETA- HadGEM2-ES tem suas condições de contorno atualizadas a cada 6 horas, com resolução espacial de 20 km e 38 níveis verticais, cobrindo todo o território brasileiro, além de grande parte da América do Sul e Central [9].

Foram utilizados no estudo os dados históricos de precipitação $(\mathrm{mm})$ em escala diária do RCM e selecionadas as séries de campo para as três regiões climáticas do estado de Sergipe. A baixa cobertura existente no Estado com postos de séries históricas longas mostra-se como dificuldade para estudos neste tema. Assim buscou-se avaliar dados espacializados disponíveis que permitissem a avaliação considerando as áreas estudadas na forma de grades. Esta primeira avaliação fez uso de dados de campo de sete estações pluviométricas espalhadas pelo estado, com séries históricas acima de 20 anos de dados, obtidas no portal Hidroweb da Agência Nacional de Águas [12] e foi verificada a concordância entre os seus dados mensais e os dados extraídos na mesma localização espacial a partir do produto Brazil Gridded Meteorological Data (BGMD) [13], que possui resolução espacial de $0,25^{\circ}$ e cobertura sobre todo o território nacional. Este produto pode ser acessado em https://utexas.app.box.com/v/xavier-etal-ijoc-data.

O grau de precisão da estimativa foi avaliado por meio da consideração de dois critérios de performance: coeficiente de correlação de Pearson (r) e a Raiz do Erro Médio Quadrático (RMSE).

$\mathrm{Na}$ estatística descritiva o coeficiente de correlação de Pearson (r) representa o grau de correlação, positiva ou negativa entre duas amostras, variando de -1 a 1 . 
Tabela 1: Estações pluviométricas utilizadas no estudo no estado de Sergipe [12]

\begin{tabular}{llrrr}
\hline Código & Nome & Latitude & Longitude & Altitude (m) \\
\hline 1037004 & ARACAJU & $-10,917$ & $-37,050$ & 4,72 \\
1037078 CAPELA & $-10,483$ & $-37,067$ & 139,00 \\
1137017 & ESTÂNCIA & $-11,267$ & $-37,443$ & 53,00 \\
1037007 & FAZENDA BELÉM & $-10,942$ & $-37,345$ & 19,00 \\
1036048 & PROPRIÁ & $-10,215$ & $-36,824$ & 17,00 \\
1137001 & SALGADO & $-11,030$ & $-37,478$ & 92,00 \\
1037049 & SANTA ROSA DE LIMA (CAMBOATA) & $-10,653$ & $-37,193$ & 47,00 \\
\hline
\end{tabular}

$$
r=\frac{\sum_{i=1}^{n}\left(Y_{i}-\bar{Y}\right)\left(X_{i}-\bar{X}\right)}{\sqrt{\sum_{i=1}^{n}\left(Y_{i}-\bar{Y}\right)^{2}} \cdot \sqrt{\sum_{i=1}^{n}\left(X_{i}-\bar{X}\right)^{2}}}
$$

Associado ao coeficiente de correlação, foi avaliada a condição de similaridade das séries por meio da aplicação do teste t-Student ao nível de significância de 0,05 .

A Raiz do Erro Médio Quadrático (RMSE), é normalmente usada para avaliar a precisão das estimativas com relação a dados observados, apresentado valores de erro nas mesmas dimensões da variável.

$$
R M S E=\sqrt{\frac{\sum_{i=1}^{n}\left(Y_{i}-X_{i}\right)^{2}}{n}}
$$

onde X e Y representam, respectivamente, os valores de precipitação mensal obtidos nas estações pluviométricas e na grade do BGMD nas mesmas coordenadas das estações. O número de registros mensais é representado por $n$.

Desta forma, verificada a condição adequada de representação do comportamento das chuvas no Estado pelo produto BGMD, foram extraídos deste produto as precipitações mensais nas mesmas coordenadas da grade do ETA. Assim, foram obtidas duas séries históricas de trinta anos: campo (do BGMD) e ETA. A avaliação da representatividade do ETA sobre o comportamento das chuvas no Estado também fez uso dos critérios acima citados: $r$ e RMSE.

Para extração e manipulação de todos os dados foram desenvolvidos scripts no software livre $\mathrm{R}$ v.3.3.1 [14].

\section{RESULTADOS E DISCUSSÃO}

A verificação da condição das séries de precipitação obtidas do produto BGMD representarem adequadamente o comportamento sazonal das chuvas no estado de Sergipe foi realizada a partir da análise dos indicadores de performance para os sete postos pluviométricos existentes. Pode ser observado na Tabela 2 os valores dos coeficientes obtidos para os postos. Verifica-se que, de forma geral, os dados extraídos do BGMD representaram muito bem as chuvas mensais nos postos, com o coeficiente de correlação variando de 0,94 (estação Salgado) a 0,99 (estação Fazenda Belém).

Os valores de RMSE também foram satisfatórios, variando de 16,21 mm, para a estação Fazenda Belém, a 33,70 mm, para Salgado. A partir dos gráficos apresentados na Figura 2, observa-se que que estas diferenças apontadas pelo RMSE ocorreram nos meses chuvosos, representando, em percentuais, algo em torno de 3,0 a 8,0\% em média dos valores no período úmido. Nos meses com menos chuva as diferenças obtidas foram muito baixas.

Considerando os valores obtidos para a probabilidade (p), observa-se que em todos os postos não há indicativo de diferenças significativas entre as séries avaliadas, tendo em vista que todos 
foram superiores ao nível de significância adotado $(\mathrm{p}>0,05)$. Tais resultados apontam para uma adequada representação da sazonalidade das chuvas nas diferentes regiões climáticas do Estado de Sergipe pelo produto BGMD.

Tabela 2: Valores dos coeficientes de performance obtidos para as séries de precipitação das estações pluviométricas e do produto Brazil Gridded Meteorological Data (BGMD) para o estado de Sergipe

\begin{tabular}{lccc}
\hline Estação pluviométrica & R & p & RMSE \\
\hline ARACAJU & 0,96 & 0,337 & 27,53 \\
CAPELA & 0,98 & 0,273 & 19,07 \\
ESTÂNCIA & 0,98 & 0,140 & 22,80 \\
FAZENDA BELÉM & 0,99 & 0,506 & 16,21 \\
PROPRIÁ & 0,97 & 0,220 & 17,04 \\
SALGADO & 0,94 & 0,357 & 33,70 \\
SANTA ROSA DE LIMA (CAMBOATA) & 0,98 & 0,232 & 18,30 \\
\hline
\end{tabular}

$\mathrm{r}$ - coeficiente de correlação de Pearson; $\mathrm{p}$ - probabilidade associada ao r; RMSE - Raiz do Erro Médio Quadrático

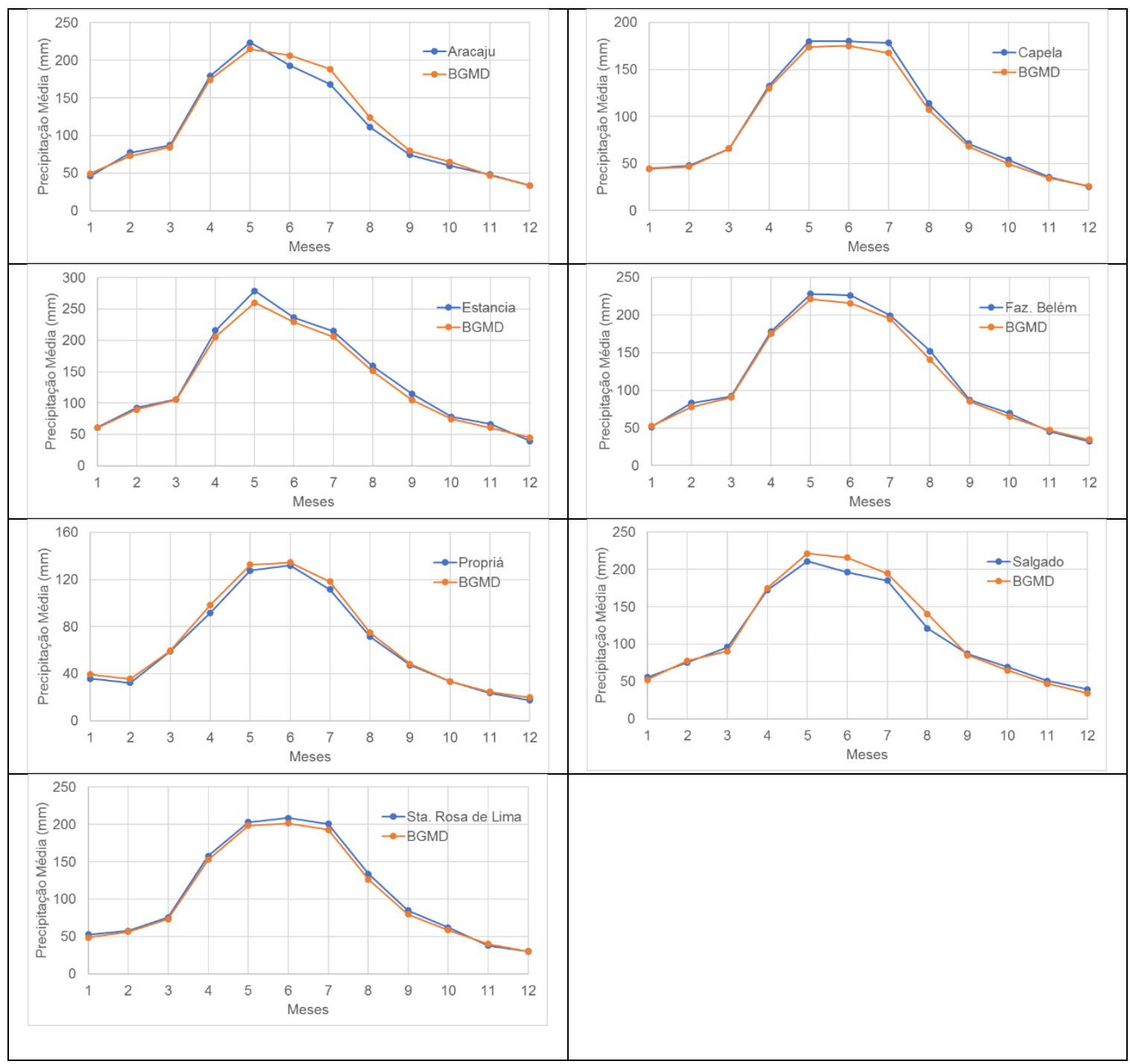

Figura 2: Precipitações médias mensais para os sete postos selecionados no estado de Sergipe em comparação aos valores médios extraídos do produto Brazil Gridded Meteorological Data (BGMT)

Uma vez que os resultados apontam para a viabilidade de utilização do BGMD como base de referência, sobre todo o território do Estado de Sergipe, este foi considerado para análise 
comparativa com os valores médios mensais históricos de modelos de mudanças climáticas. Assim, foram extraídas do BGMD as séries de precipitações referentes às coordenadas de localização de todos os pontos da grade do ETA sobre o estado de Sergipe e avaliada a performance por meio dos coeficientes já citados.

Na Tabela 3 estão apresentados os valores dos coeficientes para a análise comparativa entre as séries de precipitação nas três regiões climáticas considerando os dados do BGMD e do ETA. Observa-se que os valores dos coeficientes obtidos indicam muito baixa capacidade de representação da sazonalidade de forma adequada nas três regiões climáticas do Estado de Sergipe. Os valores do coeficiente de correlação de Pearson (r), situaram-se entre 0,019 e 0,143 , não indicando, portanto, a existência de correlação significativa. Na região Semiárida houve uma pequena melhora na correlação, no entanto, os valores ainda foram muito baixos, com valor médio de 0,102 .

Tabela 3: Valores dos coeficientes de performance obtidos para as séries de precipitação do produto Brazil Gridded Meteorological Data (BGMD) e histórico do modelo ETA-HagGEM2 para o estado de Sergipe

\begin{tabular}{ccccccccc}
\hline \multirow{2}{*}{ Região Climática } & N. pontos & \multicolumn{3}{c}{$\mathrm{r}$} & \multicolumn{2}{c}{$\mathrm{p}$} & \multicolumn{3}{c}{ RMSE } \\
& & Min. & Med. & Max. & & Min. & Med. & Max. \\
\hline Litoral Úmido & 11 & 0,019 & 0,059 & 0,088 & $<<0,05$ & 129,20 & 137,49 & 143,80 \\
Agreste & 15 & 0,020 & 0,052 & 0,102 & $<<0,05$ & 111,20 & 123,89 & 136,70 \\
Semiárido & 20 & 0,043 & 0,102 & 0,143 & $<<0,05$ & 58,70 & 74,34 & 93,80 \\
\hline
\end{tabular}

Para todos os pontos da grade avaliados em todas as regiões, a probabilidade $\mathrm{p}$ sempre apresentou valores inferiores ao nível e significância adotado $(0,05)$, apontando para indicativos de diferenças significativas entre as séries históricas comparadas. A interpretação anterior é reforçada também pelos altos valores obtidos para o coeficiente RMSE, variando de $58,70 \mathrm{~mm}$ na região Semiárida a 143,80 mm no Litoral Úmido. A dificuldade de representação da sazonalidade no Estado de Sergipe pelo modelo regional deve estar associada à complexidade dos sistemas climáticos que atuam na faixa litorânea do Brasil [10] e também às condições de contorno da versão do RCM utilizada neste estudo, que usa saídas do modelo global ETA-HadGEM2, o qual teve também os dados de precipitação históricos avaliados pelos autores e percebeu-se comportamento similar ao do ETA, logicamente com problemas também decorrentes da dimensão da escala. Na Figura 3 estão apresentados os gráficos médios da sazonalidade nas três regiões climáticas avaliadas.

Observa-se pela Figura 3, que os dados ETA indicam em todas as regiões volumes totais médios anuais inferiores aos registrados, sendo que para o Litoral Úmido, o volume anual estimado pelo RCM foi da ordem de 74\% do volume fornecido pelo BGMD, para o Agreste esta relação ficou em $61 \%$ e para o Semiárido, $39 \%$. Ademais à subestimação dos volumes precipitados anuais, verificase também que o RCM não consegue fornecer uma representação perfeita da sazonalidade nas três regiões do Estado, deslocando os meses de maiores alturas pluviométricas para março-abril-maio, reduzindo significativamente os volumes de chuva nos meses de maio, junho e julho, que concentram o período chuvoso no estado de Sergipe e imediações. Segundo Chou et al. (2005) [17] o ETA, quando aninhado ao CPTEC/COLA, mantinha alguns erros deste, com maiores magnitudes localizadas geograficamente ao longo da costa norte e nordeste do Brasil e sobre os Andes. Não foram encontrados estudos com esta avaliação nas costas brasileiras para esta versão do ETA HadGEM2-SE. Estudos apontam que a previsão de chuva sobre o Nordeste do Brasil, principalmente nos meses de fevereiro a abril, depende da anomalia de temperatura da superfície do mar (TSM) sobre o Atlântico tropical. As maiores falhas nestas previsões ocorrem quando as anomalias de TSM, sobre o Atlântico tropical norte, são grandes e não correlacionadas com os padrões de anomalia sobre o Oceano Pacífico leste[18]. 

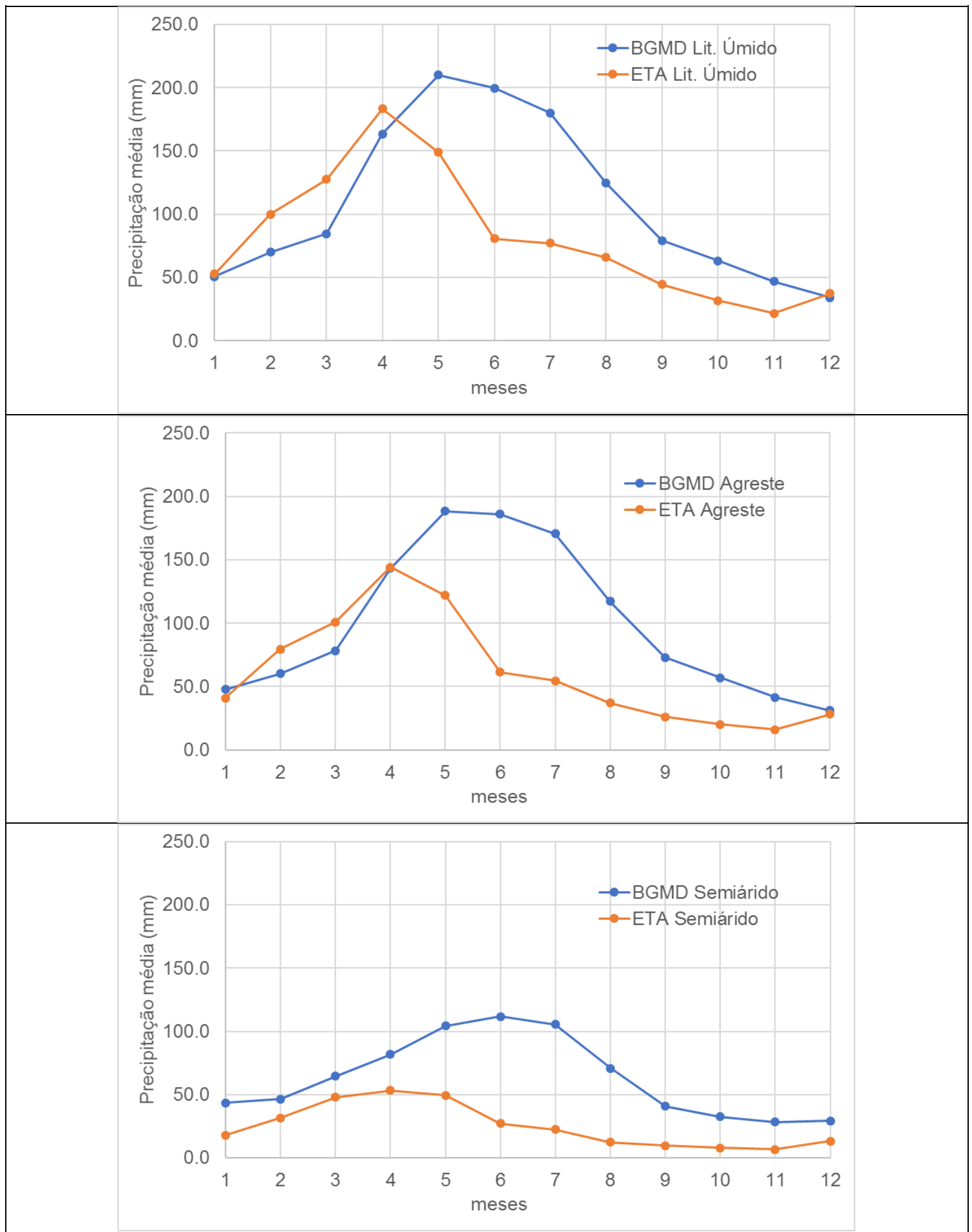

Figura 3: Precipitações médias mensais para as três regiões climáticas no estado de Sergipe obtidas do produto Brazil Gridded Meteorological Data (BGMT) e do RCM ETA-HadGEM2-ES. 


\section{CONCLUSÃO}

Os resultados obtidos no estudo apontam para uma baixa capacidade de representação da sazonalidade intra-anual por parte do RCM ETA HadGEM2-ES nas três regiões climáticas existentes no estado de Sergipe, uma vez que os valores dos coeficientes de correlação de Pearson para as chuvas mensais foram muito baixos, variando de 0,019 a 0,143 e a raiz do erro médio quadrático, mostrou-se bastante elevada, com variação entre 58,7 e 143,8 mm. Tais resultados são reflexo de que o modelo subestimou os volumes precipitados nas regiões entre 39 e $74 \%$, indicando o período chuvoso concentrado de março a maio, quando os maiores volumes ocorrem na região entre os meses de maio e julho.

Este comportamento provavelmente está associado às condições de contorno consideradas pelo ETA, provenientes do modelo global HadGEM2-ES, que por sua vez indica, considerando a sua resolução espacial menos refinada, concentração de chuvas mais no início do ano nas células que cobrem esta região.

\section{AGRADECIMENTOS}

À FAPITEC/SE pela cedência da bolsa PIBIC ao aluno que desenvolveu o estudo.

\section{REFERÊNCIAS BIBLIOGRÁFICAS}

1. PBMC. Painel Brasileiro de Mudanças Climáticas. Mudanças ambientais de curto e longo prazo: projeções, reversibilidade e atribuição. In Primeiro Relatório de Avaliação Nacional. Brasília. 2016;320346.

2. Watanabe S, Kanae S, Seto S, Yeh PJ-F, Hirabayashi Y, Oki T. Intercomparison of bias-correction methods for monthly temperature and precipitation simulated by multiple climate models. J Geophys Res. 2012;117:D23114, doi:10.1029/2012JD018192.

3. Maraun D, Wetterhall F, Ireson AM, Chandler RE, Kendon EJ, Widmann M, Brienen S, Rust HW, Sauter T, Themel M, Venema VKC, Chun KP, Goodess CM, Jones RG, Onof C, Vrac M, Thiele-Eich I. Precipitation downscaling under climate change: Recent developments to bridge the gap between dynamical models and the end user. Rev Geophys. 2010;48:RG3003, doi:10.1029/2009RG000314.

4. Veljovic K, Rajkovic B, Fennessy MJ, Altshuler EL, Mesinger F. Regional climate modeling: Should one attempt improving on the large scales? Lateral boundary condition scheme: Any impact? Meteor Z. 2010 Jun;19(3):237-246, doi:https://doi.org/10.1127/0941-2948/2010/0460.

5. Chou SC, Nunes AMB, Cavalcanti IFA. Extended range forecasts over South America using the regional Eta Model. J Geophys Res. 2000 Apr;105(D8):10147-10160, doi:10.1029/1999JD901137.

6. Nobre P, Moura AD, Sun L. Dynamical downscaling of seasonal climate prediction over Northeast Brazil with ECHAM3and NCEP's Regional Spectral Models at IRI. Bull Amer Meteor Soc 2001 Dec;82(12):2787-2796, doi:10.1175/1520-0477(2001)082<2787\%3ADDOSCP>2.3.CO\%3B2.

7. Chou SC, Marengo JA, Lyra A, Sueiro G, Pesquero JF. Downscaling of South America present climate driven by 4-member HadCM3 runs. Clim Dyn. 2012 Feb;38(3-4):635-653, doi:10.1007/s00382-0111002-8.

8. Fernandez JPR, Franchito SH, Rao VB. Simulation of the summer circulation over South America by two regional climate models Part I Mean climatology. Theor Appl Climatol. 2006 Sep;86:247-260, doi:10.1007/s00704-005-0212-6.

9. Chou SC, Lyra A, Mourão C, Dereczynski C, Pilotto I. Evaluation of the Eta Simulations Nested in Three Global Climate Models. American J Climate Change 2014 Dec;3:438-454, doi:10.4236/ajcc.2014.35039.

10. Ferreira AG, Mello NGS. Principais sistemas atmosféricos atuantes sobre a região Nordeste do Brasil e a influência dos oceanos Pacífico e Atlântico no clima da região. Rev Bras Climatol. 2005 Dez;1(1):15-28, doi:10.5380/abclima.v1i1.25215.

11. SERGIPE. Caracterização Climática. Portal do Governo de Sergipe. Secretaria de Estado do Meio Ambiente e dos Recursos Hídricos. Disponível em: < https:// http://www.semarh.se.gov.br/meteorologia/>. Acesso em: Mar. 2017.

12. ANA. Sistemas de Informações Hidrológicas - HIDROWEB. Agência Nacional de Águas. 2017. Disponível em: http://www.hidroweb.ana.gov.br/. Acesso em: 03 abr. 2017. 
13. Xavier AC, Kingb CW, Scanlonc BR. Daily gridded meteorological variables in Brazil (1980-2013). Int J Climatol. 2016 May;36(6):2644-2659, doi:10.1002/joc.4518.

14. R Core Team. R: A Language and Environment for Statistical Computing. Version 3.2.2. R Foundation for Statistical Computing. Vienna, Austria. Disponível em: < https://www.R-project.org>. Acesso em: Mar. 2017.

15. Sun L, Moncunill DV, Li H, Moura AD, de Souza FA. Climate Downscaling over Northeast of Brazil, using the NCEP RSM97. J Climate. 2005 Feb;18:551-567, doi:10.1175/JCLI-3266.1.

16. Machado RD, Rocha R P. Previsões climáticas sazonais sobre o Brasil: avaliação do RegCM3 aninhado no modelo global CPTEC/COLA. Rev Bras Meteor. 2011;26(1):121-136.

17. Chou SC, Bustamante JF, Gomes JL. Evaluation of Eta Model seasonal precipitation forecasts over South America. Nonlinear Processes in Geophysics 2005 Jun;12:537-555, doi:hal-00302616.

18. Misra V. Understanding the predictability of seasonal precipitation over northeast Brazil. Tellus 2006 May;58:307-319, doi:10.1111/j.1600-0870.2006.00175.x 